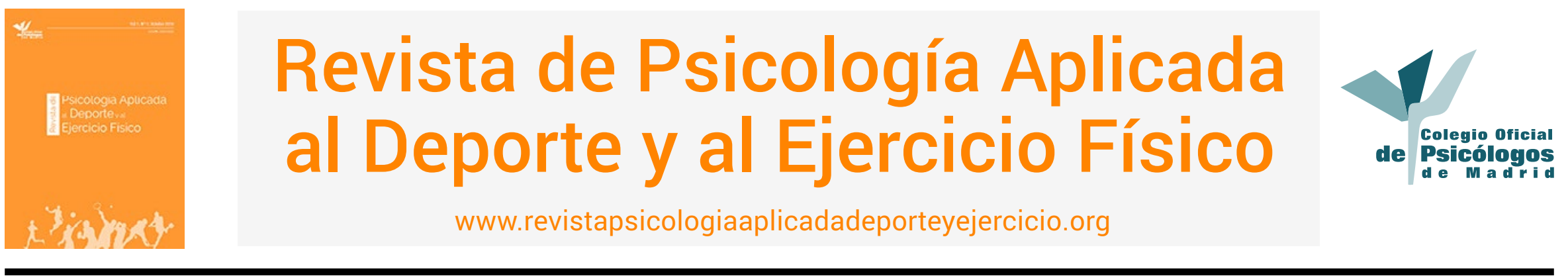

\title{
Beneficios cognitivos, psicológicos y personales del uso de los videojuegos y esports: una revisión
}

\author{
Alejo García-Naveira Vaamondea, Martin Jiménez Toribio ${ }^{b}$, Borja Teruel Molero $^{c}$ y Alejandro Suárez ${ }^{c}$ \\ ${ }^{a}$ MAD Lions E.C \\ ${ }^{\mathrm{b}}$ Freelance \\ c Universidad de Valencia
}

RESUMEN: El objetivo del presente trabajo es realizar una revisión de estudios sobre los beneficios cognitivos, psicológicos y personales del uso de los videojuegos y esports. Para ello, se utilizaron las bases de datos Google Académico y Psicodoc. El volumen total de referencias fue de 51.800 documentos, publicados entre los años 2012 y 2018, de los que se seleccionaron 26 trabajos representativos sobre la temática. Los resultados de la revisión tienden a indicar que el empleo regular de los videojuegos y esports mejoran las capacidades cognitivas, habilidades psicológicos y el desarrollo de valores de las personas. Se concluye que los videojuegos y los esports pueden ser una herramienta útil para el entrenamiento y evaluación mental, en el que queda por definir las horas diarias óptimas de uso para su beneficio y la necesidad de compaginar con otras actividades (estudios, actividad física, hábitos de descanso...), retos a abordar dentro de la Psicología del Deporte.

PALABRAS CLAVE: gamer, esports, videojuegos y Psicología del Deporte.

\section{Cognitive, psychological, and personal benefits of the use of video games and e-sports: a review}

ABSTRACT: The aim of this study was to review studies on the cognitive, psychological, and personal benefits of the use of video games and e-sports. For this, Google Academic and Psicodoc databases were used. The total volume of references was 51,800 documents published between 2012 and 2018, of which 26 representative reports on the subject were selected. The results of the review tend to suggest that regular use of video games and e-sports improves cognitive abilities, psychological skills, and development of values in people. It is concluded that videogames and e-sports may be a helpful tool for mental training and evaluation. The optimal daily time of use to achieve benefit and the need to combine these with other activities (studies, physical activity, rest ...) must still be defined, and represent challenges to be addressed by sport psychology.

KEYWORDS: Gamer, e-sports, videogames, sports psychology.

\section{Benefícios cognitivos, psicológicos e pessoais do uso de videojogos e desportos eletrónicos: uma revisão}

RESUMO: O objetivo deste trabalho é realizar uma revisão de estudos sobre os benefícios cognitivos, psicológicos e pessoais do uso de videojogos e desportos eletrónicos. Para isso, foram usadas as bases de dados Google académico e Psicodoc. O volume total de referências foi de 51.800 documentos, publicados entre 2012 e 2018, dos quais foram selecionados 26 trabalhos representativos sobre o tema. Os resultados da revisão tendem a indicar que o uso regular de videojogos e desportos eletrónicos melhoram as competências cognitivas, psicológicas e o desenvolvimento de valores das pessoas. Conclui-se que os videojogos e os desportos eletrónicos podem ser uma ferramenta útil para o treino e avaliação mental, sendo que resta definir as melhores horas diárias de uso para seu benefício e a necessidade de se conciliar com outras atividades (estudos, atividade física, hábitos de descanso ...), desafios a serem abordados na Psicologia do desporto.

\footnotetext{
Alejo García-Naveira Vaamonde. Es coordinador Área de Psicología del Deporte e I+D+i de MAD Lions E.C.; Martin Jiménez Toribio. Es psicólogo, Freelance: Borja Teruel Molero. Es estudiante de psicología de la Universidad de Valencia; Alejandro Suárez. Es licenciado en Educación Física. Responsable del área de Rendimiento de MAD Lions E.C.
} 
PALAVRAS-CHAVE: jogador, desportos eletrónicos, videojogos e psicologia desportiva.

Artículo recibido:-06/08/2018 | Artículo aceptado: 30/10/2018

Durante los últimos años, impulsados por el creciente avance tecnológico y la conectividad en red, los videojuegos (juego electrónico que se visualiza en una pantalla, por ejemplo, a través de ordenadores, móviles o videoconsolas) y esports (deportes electrónicos, donde se compite en determinados videojuegos) son una realidad social, económica, laboral y de entretenimiento, que vive una expansión a nivel mundial (DEV, 2016; Hamari y Sjöblom, 2017; Stanton, 2015). A modo de ejemplificar esta afirmación, la industria del videojuego en el año 2017 fue capaz de generar a nivel mundial 116.000 millones de dólares y hubo 2.200 millones de personas que jugaron a algún videojuego (Newzoo, 2017). Por su parte, los esports en el año 2017 generaron un ingreso de 655 millones de dólares y hubo 143 millones de espectadores a nivel mundial (Newzoo, 2018).

Los esports surgen de la actividad reglada, competitiva y profesional de determinados videojuegos, siendo estos últimos jugados por ocio y recreación (Bányai, Griffiths, Király y Demetrovics, 2018; Hamari y Sjöblom, 2017; Taylor, 2012). Actualmente, existen competiciones nacionales e internacionales organizadas por diferentes entidades (p. ej., la Liga de Videojuegos Profesional en España) en formato de liga, copa o evento que se juegan a lo largo de una temporada deportiva. Los principales videojuegos en los cuales se compite mundialmente a nivel individual o en equipo, son, entre otros: League of Legends, Dota 2, Counter Strike, FIFA o Call of Duty (DEV, 2016).

Independientemente de si los esports pueden ser clasificados como deporte, cuestión controvertida y de escasa importancia práctica (Funk, Pizzo y Baker, 2018; Hallmann y Giel, 2018; Sánchez y Remilllard, 2018), reúne las características propias de los deportes tradicionales, aspectos que si son relevantes desde lo aplicable y su estudio (ONTIER 2018): (i) hay un rendimiento principalmente mental, aunque en menor medida, también físico; (ii) la participación es organizada; (iii) existe competiciones regladas; (iv) hay entrenamientos; (v) están sujetos a normas y (vi) tiene un carácter competitivo.

Desde una perspectiva del rendimiento, diferentes autores (Bányai et al., 2018; Hilvoorde y Pot, 2016; Kari y Karhulahti, 2016; Railsback y Caporusso, 2019; Taylor, 2012) definen a los esports como un tipo de deporte donde las personas desarrollan y entrenan las capacidades cognitivas (p. ej., tiempo de reacción y toma de decisiones), habilidades mentales ( $p$. ej., motivación y autoconfianza), capacidades físicas (p. ej., la resistencia para contrarrestar la fatiga, ya que algunas eliminatorias o partidos pueden durar entre 2-4 horas seguidas), lo técnico-táctico (p. ej., la estrategia), la psicomotricidad ( $p$. ej., óculo-manual) y los valores tradicionales (p. ej., esfuerzo y superación), con el uso de las tecnologías.

Por ejemplo, una persona puede jugar al FIFA (juego de fútbol) como un videojuego (p. ej., jugando los fines de semana con sus amigos), pero la organización de competiciones (ligas y torneos con premios establecidos) y la preparación del participante para competir, hace que la actividad se profesionalice (p. ej., entrenando un total de 4-6 horas diarias durante 5-6 días a la semana).

Varios de estos esports se integran en clubes profesionales constituidos legalmente con jugadores, staff técnico y directiva, generando diferentes puestos de trabajo -entrenadores, preparadores físicos, psicólogo del deporte, nutricionistas, fisioterapeutas, asesores de imagen, youtubers, marketing, publicidad, etc.- (p. ej., ver MAD Lions E.C., 2018). Estos clubes buscan una productividad empresarial y un desempeño deportivo, con las consiguientes retransmisiones de las competiciones -p. ej., Twitch- (Gerber, 2017).

Partiendo del alto impacto que tienen los videojuegos y esports, en los últimos años ha habido un aumento del número de investigaciones que estudian la influencia de estos sobre las personas (Billieux, Deleuze, Griffiths y Kuss, 2015; Zhong, 2011). Aunque es bastante estudiado los efectos negativos comportamentales de las nuevas tecnologías sobre los sujetos (adicción, bajo rendimiento académico, tiempo perdido, inhibición, etc.), actualmente existe una línea de investigación positiva entorno a los beneficios de su uso, menos conocida, que requiere seguir siendo estudiada, pero de alto interés social y educativo (Nuyens, Kuss, Lopez-Fernández y Griffiths, 2017). Por ejemplo, estos autores, destacan el uso de los videojuegos dentro de programas para la mejora de la calidad de vida y como acción terapéutica en la promoción de la salud (p. ej., uso de drogas y el síndrome de inmunodeficiencia adquirida). Por su parte, González-González, del Río y Navarro-Adelantado (2018) concluyen que la personalización de la experiencia del usuario y las tecnologías emergentes (big data, wearables, tecnologías inteligentes, etc.) mejoran la práctica deportiva y la motivación entre los jóvenes, y se presentan como oportunidades prometedoras para mantener el compromiso y el ejercicio físico a través de programas basados en el videojuego y las nuevas tecnologías. 
Partiendo de estas premisas, dado que los videojuegos y esports tienen un marcado carácter mental, el objetivo del presente trabajo es realizar una revisión de trabajos sobre los beneficios positivos que producen sobre la persona, en concreto, en relación a las capacidades cognitivas, habilidades psicológicas y desarrollo de valores.

\section{Método}

Para llevar a cabo este trabajo, se han utilizado como fuentes de búsqueda, la información disponible en las siguientes bases de datos: Google Académico y Psicodoc, desde el año 2012 al 2018, utilizando como palabras clave "Psychology", asociado con otros términos relacionados con los videojuegos y el sector (esport, video game y meta, video game y review, burnout, CSGO, counter strike global offense, LOL, League of Legends, overwatch, rainbow six siege, hots, heroes of the storm, Hearthstone, Starcraft 2, WOW, World of Warcraft, Halo, FPS, First Person Shooter, MOBA, MMORPG y Strategy), limitando los términos de búsqueda al título, resumen y palabras clave.

El volumen total de referencias fue de 51.800 documentos (octubre de 2018), de los que se seleccionaron 26 trabajos. Como criterios, se estableció seleccionar solo aquellos que reuniera las siguientes condiciones: a) tienen que provenir de revistas o congresos científicos-profesionales, b) la temática tienen que abordar los beneficios comportamentales del uso de los videojuegos y/o esports, excluyendo los estudios sobre trastornos o psicopatologías y c) los documentos tienen que integrar alguno de los siguientes géneros de videojuegos, debido a que son los que más gente lo práctica (ver Gamerdic, 2018): Acción en primera persona (First Person Shooter), Multijugador online (Massive Online Battle Arena), MMORPG (Massively Multiplayer Online Role-Playing Game) y Estrategia.

\section{Revisión de investigaciones}

La hipótesis bidireccional de influencia entre la personalidad del individuo y la práctica de la actividad física y el deporte, indica que, en la interacción gen (herencia) y ambiente (actividad), ciertas características y habilidades psicológicas pueden facilitar el rendimiento deportivo, mientras que la propia actividad física y el deporte influye en el desarrollo de la persona (Allen, Greenless y Jones, 2013; Allen, Magee, Vella, y Laborde, 2016; Ruiz-Barquín y García-Naveira, 2013), cuestiones que ya se han percibido en los esports (Pérez-Rubio, González y Garcés de los Fayos, 2017).

Desde esta perspectiva, el uso regular de los videojuegos y los esports producen una estimulación cerebral, activando y desarrollando diferentes estructuras de la misma en los individuos (Adamos et al., 2017; Hyun et al., 2013; Song, Han y Shim, 2013). Como se verá posteriormente, esta actividad representa un medio para la mejora de capacidades cognitivas, habilidades psicológicas y desarrollo de valores, cuestiones que le dan estructura al presente apartado. A continuación, se presenta la revisión de trabajos sobre beneficios psicológicos de los videojuegos y esports.

Capacidades cognitivas. Históricamente, la influencia de los esports y videojuegos sobre las capacidades cognitivas de las personas ha sido el principal foco de interés entre los investigadores (Meinz et al., 2012), observándose una mejora en general en función de las variables estudiadas.

Colzato, van den Wildenberg, Zmigrod y Hommel (2012) obtienen que, en general, el uso de los videojuegos mejora la memoria de trabajo, la flexibilidad cognitiva y la búsqueda visual de los usuarios. Chiappe, Conger, Liao, Caldwell, y Vu (2013) señalan que los videojuegos, concretamente de acción, mejoran la habilidad multitarea (habilidad para procesar múltiples elementos informativos interactuando mientras se llevan a cabo varias subtareas), la habilidad para procesar información periférica y la habilidad para procesar la comunicación verbal. Powers, Brooks, Aldrich, Palladino, y Alfieri (2013) concluyen en su meta-análisis que, en general, la práctica regular de los videojuegos mejoran el procesamiento de la información (procesamiento auditivo, funciones ejecutivas, habilidades motoras, imágenes espaciales y procesamiento visual).

Chang, Liu, Chen y Hsieh (2017) observan que los videojuegos mejoran la capacidad de atención y el funcionamiento ejecutivo, dónde los jugadores con mayor habilidad multitarea y mayor memoria de trabajo espacial reportaron disfrutar y jugar más a juegos de Multiplayer Online Battle Arena (MOBA).

Deleuze, Christiaens, Nuyens y Billieux (2017) concluyen que el uso regular de los videojuegos mejora los tiempos de reacción de las personas. Kokkinakis, Cowling, Drachen y Wade (2017) hallaron que el rendimiento en los MOBA correlaciona con la inteligencia fluida (capacidad de un sujeto para comprender el mundo que le rodea y el conjunto de recursos con que cuenta para afrontar los retos que se le presentan) y sigue el mismo pico que el rendimiento, alcanzando su máximo potencial sobre los 25 años, con su posterior declive. Sin embargo, este declive puede verse compensado por la experiencia y las estrategias aprendidas con el videojuego como decrecer la carga cognitiva y simplificar las estrategias (Thompson, Blair, y Henrey, 2014). Bonny y Castaneda (2017) concluyen que tener una gran capacidad de procesamiento numérico aumenta el rendimiento en el videojuego, por lo que se podría suponer que si el rendimiento y el juego aumenta cuando se poseen mayor inteligencia y 
habilidades cognitivas, jugar de forma continuada puede suponer una mejora y un entrenamiento de estas capacidades (interacción sujeto-ambiente).

Richard, Mckinley y Ashley (2018) señalan que los gamers demuestran una mejora de la toma de decisiones, la reflexión y los elementos del aprendizaje individual y colaborativo. Los hallazgos ayudan a resaltar la evidencia del aprendizaje perceptivo, ya que se produce a lo largo del tiempo y mediante el perfeccionamiento de las habilidades individuales y colectivas, lo que se demuestra a través de la flexibilidad de los jugadores para adaptarse a desafíos cada vez más complejos.

Bediou et al. (2018) realizaron un meta-análisis de la influencia de los videojuegos sobre la capacidad cognitiva de las personas. Los autores concluyen que jugar con regularidad mejora la percepción, atención y la cognición espacial. En esta línea, Sala, Tatlidil y Gobet (2018) también llevaron a cabo un meta-análisis sobre la relación entre el videojuego y la capacidad cognitiva. Estos autores indican que la práctica regular de los videojuegos mejora el procesamiento visual, la atención, la capacidad de movilidad y el control cognitivo.

Destacar el trabajo de Walsh et al. (2018), que estudiaron el desempeño intelectual de 4.520 niños estadounidenses de entre 8 y 11 años en función de las recomendaciones dadas por un plan canadiense denominado Movimiento 24 horas: entre 9 y 11 horas de sueño, al menos una hora diaria de ejercicio y menos de dos horas de ocio con pantallas. Los niños estudiados completaron una prueba que evaluaba las habilidades del lenguaje, la memoria episódica, la función ejecutiva, la atención, la memoria de trabajo y la velocidad de procesamiento. Los resultados indican que cuantas más recomendaciones individuales cumplan los niños y niñas, mejores serán sus capacidades. Pero hay una pauta que destaca sobre las demás: el tiempo dedicado a los dispositivos es el que tiene una relación más fuerte con la maduración intelectual. Los autores indican que más de dos horas de tiempo recreativo con pantallas (aparatos como móviles, tabletas, ordenadores, videojuegos y también la televisión) se asocian con un peor desarrollo cognitivo en los niños.

Habilidades psicológicas. En relación a los videojuegos y esports, como se verá en los trabajos revisados, la práctica regular puede mejorar ciertas habilidades psicológicas. A nivel motivacional, diferentes investigaciones tienen como objetivo evaluar cuáles son los principales motivos que mueven a los jugadores de videojuegos. Fuster, Carbonell, Chamarro y Oberst (2013) postularon que los sujetos destacaban por la motivación por las relaciones sociales y explorar, ya que los videojuegos proveían al jugador de un espacio para experimentar y pertenecer a un grupo, dónde obtener apoyo social y diversión, alejándose de la imagen del jugador solitario que juega solo. Dindar y Akbulut (2015) obtuvieron que los gamers principalmente están motivados por el logro, seguida de la motivación de socialización, donde los jugadores con mayor autoeficacia (general y académica) tenían una mayor motivación para jugar y disfrutar de los desafíos que se encontraban en los juegos.

Otros autores defienden que otra variable importante junto a la motivación qué determina el juego y el rendimiento es la pasión armónica (la actividad ocupa un espacio importante, pero sin interferir con otros aspectos importantes de la vida), la cual influye en la motivación social, de exploración y logro de forma controlada, voluntaria y flexible (Fuster et al., 2013). En el estudio llevado a cabo por Bertran y Chamarro (2016) la pasión armónica aumentó el bienestar y la socialización. Algunos autores han reportado beneficios más concretos, como, por ejemplo, una mejora del control de la impulsividad (Puerta-Cortés, Panova, Carbonell, y Chamarro, 2017).

Gabbiadini y Greitemeyer (2017) en su estudio encontraron que los videojuegos de estrategia mejoraban capacidades psicológicas como la autorregulación, fomentando que los jugadores tuvieran que establecer metas, planificar y organizar estrategias basándose en diferentes criterios de elección, lo que resulta especialmente interesante en etapas como la adolescencia, ya que implica que estos jóvenes tengan que reflexionar acorde a unos criterios para obtener éxito, lo qué haría que los videojuegos fueran una herramienta potente en ámbitos como el educativo.

Para determinados perfiles de personalidad, el uso de videojuegos puede ser un excelente medio para expresar y desarrollar su persona. Por ejemplo, para personas con cierta ansiedad social, jugar a videojuegos de Role-Playing Game (RPG), donde tienen la posibilidad de interactuar mediante avatares personalizados en mundos virtuales, les resulta más fácil interactuar con otras personas que de forma directa cara a cara, ya que el mundo virtual ofrece una serie de herramientas donde los sujetos pueden verse protegidos y mostrarse como realmente son (Lee y Leeson, 2015). En esta línea, los videojuegos son un medio para la mejora de la competencia y la socialización en sujetos más introvertidos, en el que se espera una transferencia a la vida real (Bean, Ferro, Vissoci, Rivero y Groth-Marnat, 2016).

Por último, Carbonie, Guo y Cahalane (2018) concluyen que, a través de la práctica de los esports, los jugadores mejoran la autorregulación emocional, autoestima, iniciativa, comunicación, el crecimiento personal, logro y éxito personal, hedonismo (placer y disfrute) y propósito en la vida.

Desarrollo de valores. Los videojuegos y esports pueden ser un excelente medio para el crecimiento personal y desarrollo de valores en las personas. Se ha observado que jugar a videojuegos puede tener un papel sensibilizador ante los pro- 
blemas sociales y un impacto en el civismo (Grizzard, Tamborini, Lewis, Wang y Prabhu, 2014). Estos autores señalan que, los participantes de su estudio, tras jugar a videojuegos como terroristas, actuaron de forma más sensible ante infracciones morales y reportaron mayores sentimientos de culpa.

Jugar a videojuegos cooperativos influye en las intenciones colectivas (we-intentions) y el trabajo en equipo, siendo una forma entrenamiento que puede beneficiar el trabajo en equipo en la vida real (Morschheuser, Riar, Hamari y Maedche, 2017). Carbonie et al. (2018) destacan la mejora del compromiso, la cooperación, la recompensa por el esfuerzo y percepción de poder en los gamers.

Por su parte, Wang, Taylor y Sun (2018) estudiaron los efectos del videojuego entre los miembros de la familia sobre la satisfacción familiar y la cercanía familiar. Los resultados mostraron que mientras más frecuentemente los miembros de la familia juegan videojuegos juntos, mejor son la satisfacción familiar y la cercanía de la familia. Las familias con mala comunicación familiar se benefician más del co-juego que aquellas con comunicación familiar efectiva. La satisfacción familiar medió la relación entre el juego de video juegos y la cercanía familiar. Los participantes solían disfrutar jugando videojuegos con miembros de la familia, y los beneficios sociales son los más destacados en los entornos familiares.

Por último, destacar que en los esports pueden surgir cuestiones relacionadas con la actividad, como la deportividad (p. ej., respeto a los adversarios y cumplimiento de normas semejantes a las que deben observarse en el deporte) y los relacionados con el profesionalismo -p. ej. autosuperación, disciplina, entrenamiento y trabajo- (ONTIER 2018).

En la Tabla 1, se presenta un resumen de los trabajos revisados sobre los videojuegos y esports en relación a las capacidades cognitivas, habilidades psicológicas y desarrollo de valores.

Tabla 1. Revisión de trabajos sobre beneficios cognitivos, psicológicos y personales por el uso de los videojuegos y esports $(n=26)$

\begin{tabular}{|c|c|c|c|c|c|}
\hline Autores & Muestra & Juego & Instrumentos & Resultados & Conclusiones \\
\hline
\end{tabular}

Fuster et al. 430 sujetos (410 RPGs: Aion, DC Universe Cuestionario demo-

(2013) hombres, 20 Online, EVE Online, Lord gráfico; Playing Style mujeres) of the Rings Online, Media edad: $\quad$ Rift, World of Warcraft 26.58 años $\quad$ u otros questionnaire (estilo de juegos); Massively Multiplayer Online Games Motivations Scale, MMO-MS (motivaciones).

Multi-Attribute Task Battery, MATB (habilidad multitarea)

al. (2013)
49 individuos (24 FPS: Ghost Recon hombres y 25 mujeres) Media edad: 22 años
Advanced War Fighter 2, Unreal Tournament 3 , Medal of Honor, Vanquish, Bioshock 2 y Resistance 2
La principal motivación fue socialización seguida por un interés considerable en exploración mientras que logro era medio, puntuando bajo en disociación. Se encontraron correlaciones significativas, siendo las más marcadas entre socialización y edad, longitud de tiempo jugado y exploración y edad y logro.

Los participantes del grupo de videojuegos tuvieron menos errores y respondieron más rápidamente y mejoraron a lo largo de la sesión. Aquellos que completaban más juegos tenían mayores tiempos de respuesta y eran más consistentes en responder rápido a la comunicación. Se encontró una correlación negativa entre número de juegos completados y número de errores, mostrando un beneficio de jugar a videojuegos durante 10 semanas.
La motivación de socialización seguida de exploración fueron las más altas. En este estudio se desmiente la imagen de jugador solitario jugando RPGs, ya que se observó que el videojuego provee un espacio para experimentar y pertenecer a un grupo, para obtener apoyo social y diversión.

Los videojuegos de acción mejoraron la habilidad multitarea la habilidad para procesar información periférica y la habilidad para procesar la comunicación tras 10 semanas de uso. 


\section{Continuación Tabla 1}

\begin{tabular}{|c|c|c|c|c|}
\hline Autores & Muestra & Juego & Instrumentos & Resultados \\
\hline $\begin{array}{l}\text { Powers et al. } \\
\text { (2013) }\end{array}$ & Meta análisis & Varios videojuegos & & $\begin{array}{l}\text { La práctica regulas de los videojuegos } \\
\text { mejoran el procesamiento de la informa- } \\
\text { ción (procesamiento auditivo, funciones } \\
\text { ejecutivas, habilidades motoras, imáge- } \\
\text { nes espaciales y procesamiento visual) }\end{array}$ \\
\hline
\end{tabular}

\begin{tabular}{llll}
\hline $\begin{array}{l}\text { Fuster et al. } \\
\text { (2014) }\end{array}$ & $\begin{array}{l}\text { 410 sujetos (to- } \\
\text { dos hombres) }\end{array}$ & MMORPG: Aion-ESP, & Cuestionario demográfi- \\
& $\begin{array}{ll}\text { Media edad 26,49, JuegaEnRed, } \\
\text { años }\end{array}$ & DCUO Hispano, WoW- & la Escala de la Pasión \\
& & ESP, Guild Wars Latino, & (pasión); Massively Mul- \\
& & EVE-Online.es y Comuni- & tiplayer Online Games \\
& dad Hispana & Motivations Scale, MMO- \\
& & MS (motivaciones).
\end{tabular}

\begin{tabular}{lll}
\hline $\begin{array}{l}\text { Grizzard et } \\
\text { al. (2014) }\end{array}$ & $\begin{array}{l}185 \text { personas (63 No especifica } \\
\text { hombres, } 122\end{array}$ & Escala de 3 items de \\
& mujeres) Media & culpabilidad; Moral \\
& edad: 20.18 años & Foundations Question- \\
& naire, MFQ (atributos \\
& morales).
\end{tabular}

Thompson et 3.305 sujetos (no Starcraft 2

$\begin{array}{ll}\text { al. (2014) } & \begin{array}{l}\text { especifica sexo ni } \\ \text { edad; todo a nivel } \\ \text { telemétrico) }\end{array}\end{array}$

edad; todo a nivel

telemétrico)
En general los jugadores puntuaron más alto en pasión armónica obteniéndose relaciones significativas con motivación de exploración, socialización y logro.

\section{Conclusiones}

Los videojuegos pueden ser una actividad que mejora las capacidades cognitivas de los gamers. Se requiere mejorar los estudios a nivel metodológico.

La pasión armónica dispara de forma controlada, voluntaria y flexible, las motivaciones de socialización, exploración y logro.
Se obtuvieron correlaciones positivas entre conductas inmorales en videojuegos y culpa. Los participantes que jugaron como terroristas se sintieron más culpables que los que jugaron como soldados de UN. Los participantes que realizaron recuerdos de culpabilidad previos se sintieron más culpables al acabar

Eye-tracking (movimientos oculares y fijación en la pantalla)
La edad estaba relacionada con un incremento de latencia ver-hacer (pero el nivel de pericia no mejoró el declive) Por lo general los jugadores mayores parecen ser mejores utilizando las características de la interfaz que los jugadores jóvenes como forma de contrarrestar el declive cognitivo-motor.
Los jugadores que cometieron transgresiones morales en los videojuegos actuaron de forma más sensible ante violaciones morales. Realizar conductas atroces en entornos virtuales puede aumentar la sensibilidad ante problemas morales.

La experiencia jugando compensó el declive cognitivo que se da con la edad, decreciendo la carga cognitiva y simplificando estrategias de forma eficaz.

\begin{tabular}{|c|c|c|c|c|c|}
\hline $\begin{array}{l}\text { Dindar y Ak- } \\
\text { bulut, (2014) }\end{array}$ & $\begin{array}{l}5.380 \text { personas } \\
\text { (98,5\% hombres) } \\
\text { Edad entre 15.5- } \\
16.5 \text { años. }\end{array}$ & $\begin{array}{l}\text { MMOFPS (No especi- } \\
\text { fíca) }\end{array}$ & $\begin{array}{l}\text { Online Gaming Moti- } \\
\text { vations Questionnaire } \\
\text { (motivación); Social } \\
\text { Appearance Anxiety } \\
\text { Scale (ansiedad por } \\
\text { apariencia social); } \\
\text { General Self-Efficacy } \\
\text { Scale (autoeficacia); } \\
\text { Academic Self-Efficacy } \\
\text { Scale (autoeficacia } \\
\text { academica). }\end{array}$ & $\begin{array}{l}\text { Se obtuvieron diferencias significativas } \\
\text { entre las tres motivaciones para jugar, } \\
\text { siendo la mayor de ellas la motivación } \\
\text { de logro, seguida de motivación social } \\
\text { e inmersión. Cuando la ansiedad por } \\
\text { apariencia social se incrementó, la } \\
\text { motivación de inmersión decreció, esto } \\
\text { sugiere que jugadores de MMOFPS no } \\
\text { juegan este tipo de juegos para com- } \\
\text { pensar sus emociones negativas sobre } \\
\text { su apariencia física. }\end{array}$ & $\begin{array}{l}\text { La motivación de logro fue el } \\
\text { principal motor que movió a los } \\
\text { jugadores a jugar, seguido de } \\
\text { socialización. Alta autoeficacia ge- } \\
\text { neral y académica se relacionaron } \\
\text { con mayor motivación para jugar, } \\
\text { sugiriendo que los jugadores con } \\
\text { alta autoeficacia disfrutan de los } \\
\text { desafíos presentes en los FPS. }\end{array}$ \\
\hline $\begin{array}{l}\text { Lee y Lee- } \\
\text { son, (2015) }\end{array}$ & $\begin{array}{l}629 \text { adultos (505 } \\
\text { hombres, } 124 \\
\text { mujeres) } \\
\text { No se especifica } \\
\text { media edad }\end{array}$ & $\begin{array}{l}\text { RPG (La mayoría World } \\
\text { of Warcraft, el resto no } \\
\text { se especifica) }\end{array}$ & $\begin{array}{l}\text { Versión corta autorepor- } \\
\text { tada de Liebowitz Social } \\
\text { Anxiety Scale, LSAS- } \\
\text { SR, (ansiedad social); } \\
\text { Generalizad Problematic } \\
\text { Internet Use Scale, GPIUS } \\
\text { modificado (uso proble- } \\
\text { mático de videojuegos); } \\
\text { tiempo gastado jugando } \\
\text { (1 pregunta); cuestionario } \\
\text { creado } 4 \text { items (expre- } \\
\text { sión del self verdadero); } \\
\text { versión de } 6 \text { ítems del } \\
\text { Social Support Question- } \\
\text { naire, SSQ6 (apoyo social } \\
\text { percibido); cuestionario } \\
\text { demográfico y de juego. }\end{array}$ & $\begin{array}{l}\text { Los hallazgos sugieren que los jugado- } \\
\text { res con mayores niveles de ansiedad } \\
\text { social pueden expresar su self verda- } \\
\text { dero in-game más habitualmente que } \\
\text { en el cara a cara y reportaron mayores } \\
\text { niveles de apoyo in-game y/o menores } \\
\text { números de apoyo cara a cara. }\end{array}$ & $\begin{array}{l}\text { Los videojuegos RPG sirvieron como } \\
\text { herramienta para que los jugadores } \\
\text { con mayor nivel de ansiedad social } \\
\text { mostraran su self verdadero con } \\
\text { mayor frecuencia que en situacio- } \\
\text { nes cara a cara, obteniendo mayor } \\
\text { apoyo social in-game. }\end{array}$ \\
\hline
\end{tabular}




\section{Continuación Tabla 1}

\begin{tabular}{|c|c|c|c|c|c|}
\hline Autores & Muestra & Juego & Instrumentos & Resultados & Conclusiones \\
\hline $\begin{array}{l}\text { Bertran y } \\
\text { Chamarro, } \\
\text { (2016) }\end{array}$ & $\begin{array}{l}369 \text { individuos } \\
\text { ( } 326 \text { hombres, } 43 \\
\text { mujeres) } \\
\text { Media edad: } \\
21.59 \text { años }\end{array}$ & $\begin{array}{l}\text { League of Legends } \\
\text { (MOBA) }\end{array}$ & $\begin{array}{l}\text { Cuestionario sociode- } \\
\text { mográfico; } \\
\text { Version española de } \\
\text { la Escala de la Pasión } \\
\text { (Vallerand et al., 2003) } \\
\text { Cuestionario de Experien- } \\
\text { cias Relacionadas con } \\
\text { los Videojuegos (CERV) }\end{array}$ & $\begin{array}{l}\text { Tener una Pasión armónica por jugar } \\
\text { a videojuegos supuso menores niveles } \\
\text { de uso abusivo, pero no se han encon- } \\
\text { trado efectos en el rendimiento. }\end{array}$ & $\begin{array}{l}\text { La pasión armónica puede estar } \\
\text { positivamente relacionada con el } \\
\text { bienestar o la socialización. }\end{array}$ \\
\hline
\end{tabular}

Gabbiadini y Estudio 1: 404

Greitemeyer, personas (245

(2017) hombres, 159

mujeres)

Media edad:

30.53 años.

Estudio 2: 392

individuos (171

hombres, 221

mujeres)

Media edad:

32.03 años
Lógica, puzzles, brain training, violentos, de plataformas, simulación de carreras de coches, exergames (videojuegos activos) y de estrategia. (No especifica juegos)

Cuestionario demográfico

Bean et al. 468 sujetos (78\% World of Warcraft (RPG) (2016) hombres) Edad comprendida entre 18-25 años. $y$ de frecuencia de juego; Short Self-Regulation Questionnaire, SSRQ (autoregulación); Brief Self Control Scale (evitación de la tentación); Need for Cognition Scale (necesidad de cognición); Barrat Impulsiveness Scale (impulsividad); versión corta del Domain-Specific Risk-Taking Scale (toma de riesgos)

La cantidad de juego a videojuegos de estrategia se asoció significativamente con autorregulación.

Big Five Inventory, BFI (personalidad); cuestionario demográfico
En función del rol que tuvieron los jugadores y el tipo de servidor en el que entraron, se obtuvieron diferencias significativas en algunas dimensiones de la personalidad (mejora de la competencia y socialización en sujetos más introvertidos)

\begin{tabular}{|c|c|c|c|}
\hline $\begin{array}{l}\text { Bonny y } \\
\text { Castaneda, } \\
(2016)\end{array}$ & $\begin{array}{l}288 \text { personas } \\
\text { (274 hombres, } 14 \\
\text { mujeres) } \\
\text { Media edad: } \\
23.23 \text { años. }\end{array}$ & Dota 2 (MOBA) & $\begin{array}{l}\text { Spatial operation span } \\
\text { task (memoria de tra- } \\
\text { bajo espacial); Ordinal } \\
\text { number task (habilidad } \\
\text { de procesamiento } \\
\text { numérico). }\end{array}$ \\
\hline
\end{tabular}

Puerta-Cor- 630 sujetos (521 MMORPG, deporte, tés et al. hombres y 109 acción, simulación, (2017) mujeres Media edad: estrategia y minijuegos (no especifíca juegos) 19.53 años.

Cuestionario demográfico y de uso de videojuegos; The Passion Scale (pasión); The Impulsivity Inventory (impulsividad).

Pérez-Rubio 42 sujetos (todos League of Legends et al. (2017) hombres) (MOBA) Media edad: 19.95 años.
Cuestionario sociodemográfico (edad, estudios, posición en el juego, horas de entrenamiento, valoración resultados propios, valoración del esfuerzo y valoración de la capacidad); Inventario de Personalidad Reducido de Cinco Factores, NEOFFI (personalidad); Inventario de Burnout en Deportistas Revisado, IBD-R (burnout).
Se encontró que la habilidad cognitiva medida en un punto inicial estaba ligada con la habilidad medida 5 meses después. La habilidad cognitiva y el rendimiento y habilidad en MOBA es específico sólo en el juego individual, indicando que el contexto social influencia en los procesos cognitivos.
La frecuencia de juego a videojuegos de estrategia se asoció positivamente con autorregulación. Los videojuegos de estrategia fomentan el establecimiento de metas, planificación y elección de estrategias, lo que supone una forma de entrenamiento en autorregulación que es especialmente útil durante la adolescencia
La pasión armónica predijo la intensidad y tiempo jugando, a diferencia de la impulsividad funcional que no lo hizo.

Se obtuvieron diferencias significativas en promedio VPR (valoración de propios resultados), en el promedio VCR (valoración de la capacidad de mantener resultados), neuroticismo y cordialidad. Las relaciones directas más fuertes se encontraron entre agotamiento emocional y reducida realización personal, Las relaciones inversas más fuertes se dan entre agotamiento emocional y capacidad para mantener los resultados
Los jugadores seleccionaban roles que concordaban con su disposición individual y personalidad.

.




\section{Continuación Tabla}

\begin{tabular}{|c|c|c|c|c|c|}
\hline Autores & Muestra & Juego & Instrumentos & Resultados & Conclusiones \\
\hline $\begin{array}{l}\text { Morschheu- } \\
\text { ser et al. } \\
(2017) \text {. }\end{array}$ & $\begin{array}{l}206 \text { personas } \\
\text { (144 hombres, } 62 \\
\text { mujeres) } \\
\text { Media edad: } 34.6 \\
\text { años. }\end{array}$ & Ingress (MMOG) & $\begin{array}{l}\text { Se creó un instrumento } \\
\text { de medida tras entrevis- } \\
\text { tas con profesionales } \\
\text { y consiguiente análisis } \\
\text { paramétricos (medir } \\
\text { intenciones grupales y } \\
\text { características coopera- } \\
\text { tivas del juego Ingress) }\end{array}$ & $\begin{array}{l}\text { Utilizar características cooperativas del } \\
\text { juego estaba positivamente asocia- } \\
\text { do con todos los constructos de las } \\
\text { dinámicas de grupos, a su vez los } \\
\text { resultados indicaron que las dinámicas } \\
\text { grupales estaban asociadas con las } \\
\text { intenciones colectivas. }\end{array}$ & $\begin{array}{l}\text { Jugar a videojuegos cooperativos } \\
\text { influye en las intenciones colecti- } \\
\text { vas y al trabajo en equipo, siendo } \\
\text { una forma de cultivar y trabajar en } \\
\text { equipos que puede beneficiar el } \\
\text { trabajo en equipo en la vida real. }\end{array}$ \\
\hline $\begin{array}{l}\text { Chang et al. } \\
\text { (2017). }\end{array}$ & $\begin{array}{l}116 \text { sujetos ( } 59 \\
\text { hombres, } 57 \\
\text { mujeres) } \\
\text { Media edad: } \\
22.96 \text { años. }\end{array}$ & $\begin{array}{l}\text { MOBA y OGP (muchos } \\
\text { juegos distintos) }\end{array}$ & $\begin{array}{l}\text { Edinburgh Virtual Errands } \\
\text { Test, EVET (habilidad } \\
\text { multitarea); Working } \\
\text { Memory Index del } \\
\text { WAIS-III y del WMS-III } \\
\text { (memoria de trabajo); } \\
\text { Chen's Internet Addiction } \\
\text { Scale, CIAS (Adicción a } \\
\text { internet); Internet Usage } \\
\text { Questionnaire (uso de } \\
\text { los videojuegos); Beck } \\
\text { Depression Inventory, BDI } \\
\text { (depresión); Beck Anxiety } \\
\text { Inventory, BAI (ansieda). }\end{array}$ & $\begin{array}{l}\text { Jugar a videojuegos online estaba aso- } \\
\text { ciado con las subescalas de habilidad } \\
\text { multitarea excepto aprender y recordar. } \\
\text { Los jugadores MOBA puntuaron más } \\
\text { alto en tareas que requieren memoria } \\
\text { espacial mostrando más rápida eficien- } \\
\text { cia de navegación en el juego y seguir } \\
\text { un plan aunque las diferencias no se } \\
\text { mostraron a un nivel significativo. }\end{array}$ & $\begin{array}{l}\text { Las personas con mayor habilidad } \\
\text { multitarea y memoria de trabajo } \\
\text { espacial juegan y disfrutan más de } \\
\text { los juegos MOBA por lo que juegan } \\
\text { más. Estos descubrimientos apo- } \\
\text { yan la idea de existe una correla- } \\
\text { ción positiva entre los videojuegos } \\
\text { online y efectos en la capacidad } \\
\text { atencional y el funcionamiento } \\
\text { ejecutivo. }\end{array}$ \\
\hline $\begin{array}{l}\text { Walsh et al. } \\
\text { (2018) }\end{array}$ & $\begin{array}{l}4.520 \text { niños esta- } \\
\text { dounidenses de } \\
\text { entre } 8 \text { y } 11 \text { años. }\end{array}$ & $\begin{array}{l}\text { Diferentes tipos de } \\
\text { video juegos }\end{array}$ & $\begin{array}{l}\text { Registro de hábitos de } \\
\text { sueño, actividad física y } \\
\text { prueba que evaluaba las } \\
\text { habilidades del lenguaje, } \\
\text { la memoria episódica, } \\
\text { la función ejecutiva, la } \\
\text { atención, la memoria de } \\
\text { trabajo y la velocidad de } \\
\text { procesamiento. }\end{array}$ & $\begin{array}{l}\text { Mejor desarrollo cognitivo si se } \\
\text { cumple las pautas de sueño (entre } 9 \\
\text { y } 11 \text { horas), actividad física (al menos } \\
\text { una hora diaria) y uso de dispositivos } \\
\text { (menos de dos horas diarias) }\end{array}$ & $\begin{array}{l}\text { Hay que conseguir un equilibrio } \\
\text { entre las diferentes actividades. } \\
\text { Cuantas más recomendaciones } \\
\text { individuales cumplan los niños y } \\
\text { niñas, mejores serán sus capaci- } \\
\text { dades }\end{array}$ \\
\hline
\end{tabular}




\section{Continuación Tabla 1}

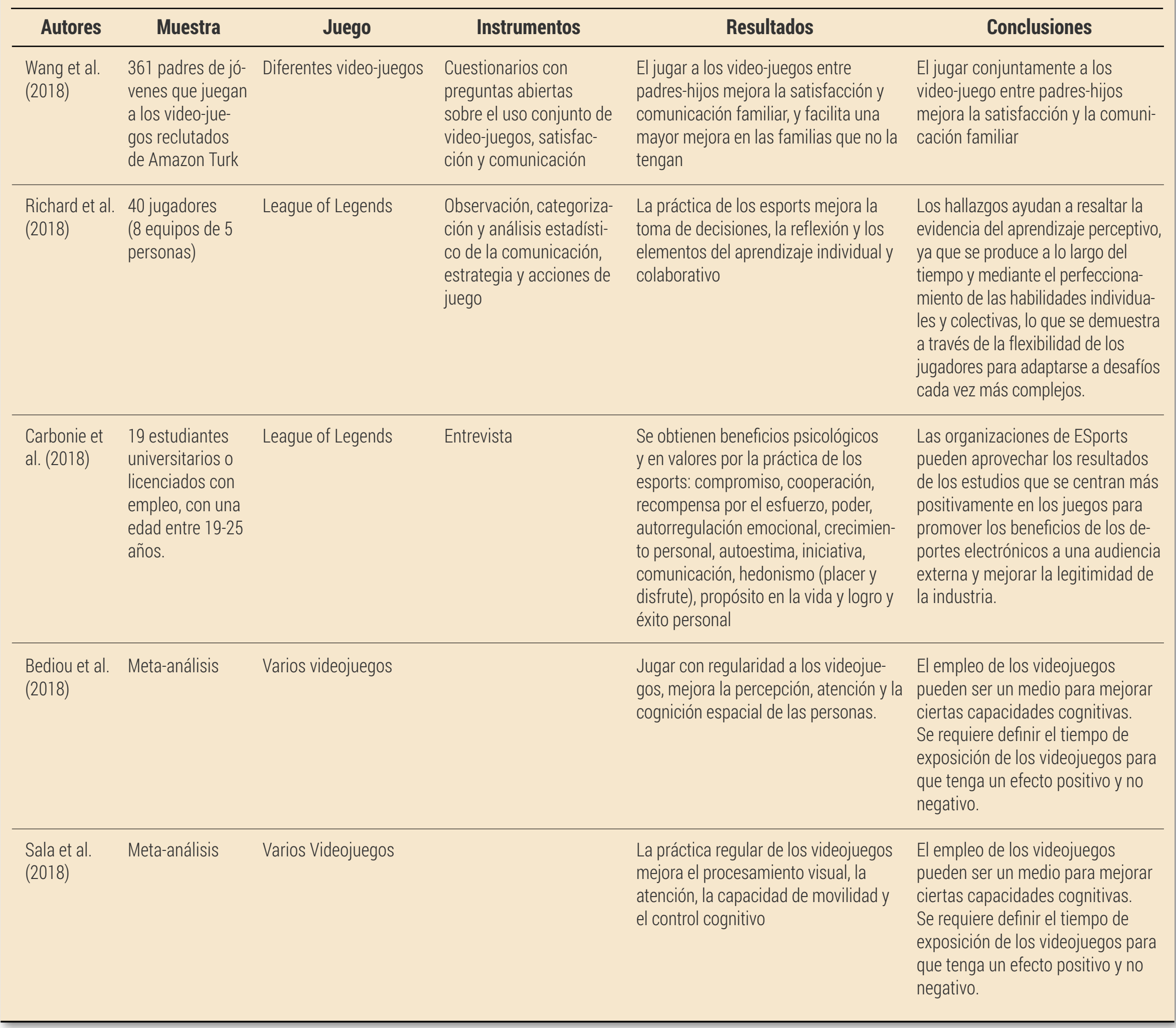

\section{Discusión}

Con la masificación de los videojuegos, el surgimiento de los esports y su profesionalización, han aumentado el interés de los investigadores por la influencia de estos sobre la persona. Desde una perspectiva positiva (Billieux et al., 2015; González-González et al., 2018; Nuyens et al., 2017), partiendo de la presente revisión, se observa que la práctica regular de los videojuegos y esports pueden producir mejoras en las capacidades cognitivas, habilidades psicológicas y en el desarrollo de valores de los individuos. A continuación, se discuten dichos resultados.
En primer lugar, indicar que, desde la hipótesis bidireccional de la personalidad (Allen et al., 2013; Allen et al., 2016; Ruiz-Barquín y García-Naveira, 2013; Pérez-Rubio et al., 2017), la persona (gen) interacciona con los videojuegos y esports (actividad/ contexto), desarrollando ciertas capacidades cognitivas, habilidades psicológicas y características de personalidad, las cuales también son necesarias para hacer frente a la tarea y tener un determinado nivel de rendimiento. Al respecto, los gamers juegan de forma regular a los videojuegos por ocio y recreación (Bányai et al., 2018; Hamari y Sjöblom, 2017; Taylor, 2012), mientras que los gamers profesionales entrenan a nivel técnico-táctico, físico y psicológico para competir en 
eventos reglados y organizados (Bányai et al., 2018; Railsback y Caporusso, 2019; Hilvoorde y Pot, 2016; Kari y Karhulahti, 2016; Taylor, 2012), cuestiones que pueden estar relacionadas con la hipótesis bidireccional de la personalidad.

En concreto, la práctica regular de los videojuegos y esports estimulan ciertas estructuras cerebrales (Adamos et al., 2017; Hyun et al., 2013; Song, Han y Shim, 2013) y mejoran las capacidades cognitivas como la inteligencia, memoria de trabajo, reflexión, toma de decisiones, flexibilidad cognitiva, búsqueda visual, habilidad multitarea, el aprendizaje procesamiento de la información y el tiempo de reacción, entre otras (Bediou et al., 2018; Bonny y Castaneda, 2017; Chang et al., 2017; Chiappe et al., 2013; Colzato et al., 2012; Deleuze et al., 2017; Kokkinakis et al., 2017; Meinz et al., 2012; Powers et al., 2013; Richard et al., 2018; Sala et al., 2018; Thompson et al., 2014; Walsh et al., 2018); posibilita el desarrollo de habilidades psicológicas como la motivación, concentración, autorregulación, autoconfianza y habilidades sociales, entre otras (Bean et al., 2016; Bertran y Chamarro, 2016; Carbonie et al., 2018; Dindar y Akbulut, 2015; Fuster et al., 2013; Gabbiadini y Greitemeyer, 2017; Lee y Leeson, 2015; Puerta-Cortés et al., 2017); y promueve el desarrollo de valores como el civismo, trabajo en equipo, compromiso, cooperación, comunicación, respeto, cumplimiento de normas, trabajo, autosuperación y disciplina, entre otras (Carbonie et al., 2018; Grizzard et al., 2014; Morschheuser et al., 2017; ONTIER 2018; Wang et al., 2018).

Como se puede observar, cada vez se encuentra mayor evidencia acerca de aspectos beneficiosos que se obtienen del uso de los videojuegos y esports, aunque no son definitivos y se requiere continuar investigando al respecto. Además, estas cuestiones no son exclusivas de este ámbito, ya que, cuando se practica con regularidad, la actividad física y el deporte tradicional pueden mejorar las capacidades cognitivas, las habilidades psicológicas y el desarrollo de valores (Cox, 2009; Weinberg y Gould, 2010).

Como indica Railsback y Caporusso (2019), lograr una mejor comprensión de los factores humanos, la dinámica y los desafíos comunes de los deportes y videojuegos profesionales, podría ayudar a definir e interpretar los deportes electrónicos y aprovechar las oportunidades que surgen de las similitudes entre los deportes tradicionales y electrónicos. La comparativa entre uno y otros puede integrar futuras líneas de investigación, aunque como se ha comprobado, el complemento entre ambas tiene un beneficio en la persona, como, por ejemplo, promocionando la actividad física y la salud entre los más jóvenes (González-González et al., 2018; Nuyens et al., 2017; Walsh et al., 2018).

Las organizaciones de esports pueden aprovechar los resultados de los estudios que se centran positivamente en los videojuegos para promover los beneficios de los deportes electrónicos a una audiencia externa y mejorar la legitimidad de la industria (Carbonie et al., 2018). Las mejoras en la legitimidad ayudan a ganar patrocinadores, ya que los patrocinadores desempeñan un papel crucial en la forma en que puede operar un equipo de esports y ayudan a potenciar el sector

Una cuestión de gran importancia a solventar es el tiempo de dedicación a los videojuegos y horas de entrenamiento en los esports, ya que en los estudios por lo general se habla de uso regular, pero no se especifica el tiempo diario y semanal que se requiere para que sea beneficioso y no llegue a ser perjudicial (Bediou et al., 2018; Deleuze, et al., 2017; Sala et al., 2018).

Cuando se trata con menores de edad, como señalan Walsh et al. (2018), se recomiendan que pediatras, padres, educadores y políticos promuevan una limitación del tiempo de pantalla recreativo (2 horas diarias), prioricen unas rutinas saludables de sueño (9-11 horas diarias) y actividad física (al menos 1 hora diaria) durante la niñez y la adolescencia. Para las familias es muy importante, porque si quieren optimizar la salud cognitiva de los hijos deberían prestar atención a estos comportamientos. Es significativo seguir investigando la influencia que tiene este tiempo de ocio en función del tipo de contenido y el uso que hacen con los menores: redes sociales, videojuegos, chatear, tareas educativas, etc.

Se requiere un equilibrio de días y horas de uso, ya que uno de los principales problemas de los videojuegos es cuando estos se utilizan de una forma irresponsable (exceso de horas al día y días de uso), en el cual la persona se evade de las tareas y los problemas de la vida, lo que puede crear una cierta dependencia psicológica (Bediou et al., 2018; Nuyens et al., 2017; Sala et al., 2018). También se ha observado en los gamers profesionales que una alta dedicación puede producir una vulnerabilidad psicopatológica (Bányai et al., 2018). Estas cuestiones se tienen que delimitar en un futuro para conseguir un beneficio positivo en el rendimiento y la salud de las personas en esports.

Otro dato importante es el que aportan Wang et al. (2018) desde una perspectiva social y educativa. El jugar a los videojuegos conjuntamente entre padres-hijos, mejora la satisfacción y la comunicación familiar. Por ello, esta línea de investigación puede ser importante desde una perspectiva de conciliación y encuentro familiar, en el que es habitual que los más jóvenes jueguen solos y sea un medio de desahogo para los padres.

\section{Aplicabilidad}

El psicólogo del deporte (Cantón, 2016) tiene ante sí una gran oportunidad laboral y profesional en la industria de los 
videojuegos y esports, para la mejora del rendimiento, la educación y el cuidado de la salud de los gamers. En concreto, los videojuegos y esports tienen un potencial para ser utilizados como entrenamiento para mejorar ciertas capacidades cognitivas, habilidades psicológicas y desarrollo de valores, siempre y cuando esté bien delimitado y gestionado a través de los entrenadores, padres, el propio jugador y diferentes profesionales del ámbito (psicólogo del deporte, preparador físico, médico, fisioterapeuta y nutricionista). Para ello, desde una perspectiva interdisciplinar, se tendría que establecer un programa de intervención que integrase estas variables y protagonistas, y, por ejemplo, realizar una evaluación pre y pos intervención, y así, observar la eficacia del mismo.

También, el psicólogo del deporte puede desarrollar escuelas de padres, entrenadores y gamers en los diferentes clubes, centros de formación o entornos académicos (colegios y universidades), para formar en actitudes, valores y conductas de uso responsable de este tipo de entretenimiento y deporte tecnológico. Además, con el crecimiento de la popularidad del sector, los videojuegos y esports son una herramienta muy útil para llegar a las poblaciones más jóvenes, lo que pueden suponer una ventaja si se utilizan y desarrollan de forma adecuada en otros ámbitos como el de la educación o la salud.

Dentro de la escuela de padres, además de promover pautas de equilibrio entre los videojuegos y esports, la actividad física, el descanso, el ocio y los estudios, sería positivo promocionar el juego familiar, cuestión vinculada a la mejora de las relaciones y comunicación entre padres, madres e hijos.

En relación a la escuela de entrenadores, se les puede adiestrar en la mejora del liderazgo y habilidades psicológicas (p. ej., motivación y comunicación) para la gestión de los gamers, el autoliderazgo (p. ej., autonomía y responsabilidad) y colaborar en el desarrollo de entrenamiento integrados (p. ej., establecer objetivos psicológicos y su gestión) y todo lo relacionado con el establecimiento y evaluación de la carga psicológica de entrenamiento dentro de una planificación deportiva. En esta línea, el entrenamiento en habilidades psicológicas para el gamer puede ser importante para afrontar con éxito la actividad (p. ej., la concentración y autorregulación emocional), así como fomentar el crecimiento personal, los estudios, hábitos saludables, hábitos de descanso, la actividad física, alimentación y el ocio.

Para finalizar, como indica Funk et al. (2018), a partir de estas cuestiones, los profesionales del ámbito deberían aprovechar el potencial de los videojuegos y esport para examinar esta evolución y proporcionar orientación a la industria a través de la educación y la investigación.

\section{Limitaciones y perspectivas futuras}

En relación a las limitaciones del presente trabajo, por un lado, destacar que es una revisión de estudios seleccionados a partir de unos criterios establecidos, por lo que puede haber otros estudios que no se hayan incluidos, por ejemplo, ampliando las bases de datos revisadas (Sport Discus, Academic Premier, Psyclit, Medline...). Además, la revisión se centra en los beneficios positivos de los videojuegos y esports, no atendiendo a los trabajos que hacen referencia a los factores negativos de su práctica, por lo que ofrecería una visión limitada. Por otro lado, no hubo una diferenciación en la revisión entre videojuegos y esports, siendo estas actividades diferenciadas, tal y como se ha definido a lo largo del presente trabajo. En futuras revisiones e investigaciones, se podría integrar ambas cuestiones (beneficios positivos y negativos) y diferenciar entre videojuegos y esports para tener una perspectiva más específica y global del asunto.

Como indican otros autores (Billieux et al., 2015; Nuyens et al., 2017; Powers et al., 2013), dentro de las limitación de estudio en este campo, se requiere un mayor rigor metodológico en relación a la constitución de la muestra (p. ej., diferenciar sexo, edad y ampliar el tamaño muestral), definir la dedicación a los videojuegos y esports (uso patológico, excesivo, determinado o casual), centrarse en los videojuegos más masificados y populares entre el público (p. ej., LOL), diferenciar los diferentes géneros del juego (p. ej., un juego de deporte como es el FIFA -fútbol- frente a uno bélico como el Call of Duty -guerra-), hacer las evaluaciones psicológicas de forma presencial (frente al online) y realizar estudios longitudinales (p. ej. 1, 2 o 3 años).

Dentro de las perspectivas futuras, la posibilidad de mejorar la capacidad cognitiva y psicológicas, así como el desarrollo en valores de las personas a través de la práctica y aprendizaje de los videojuegos, el entrenamiento y competición de los esports, son temas influyentes en la actual Psicología del Deporte y se debe de continuar investigando en próximos trabajos, superando las barreras metodológicas anteriormente citadas.

Otros temas de interés para próximos trabajos son: delimitar el número de horas óptimas al día o a la semana para el uso de los videojuegos y entrenamiento en los esports; la influencia de otras actividades (deporte, los estudios, hábitos saludables, alimentación, descanso y ocio) sobre el rendimiento y salud del gamer; analizar los proceso y variables que intervienen en el paso de un gamer amateur a otro profesional; y la influencia de los padres en el uso de los videojuegos y esports de los jóvenes.

Destacar la necesidad de que existan titulaciones oficiales para ser entrenador en esports, formación continua y re- 
ciclaje para profesionalizar su rol, garantizar un servicio de calidad y que el gamer se beneficie de los aspectos positivos revisados en el presente trabajo.

Y, por último, la Psicología del Deporte tiene grandes retos a alcanzar en los esports, como es la regulación y formación de los psicólogos que quieran trabajar en este contexto. Siguiendo lo establecido por otros autores (García-Naveira, 2018; Peris-DelCampo y Cantón, 2018), existiría unos requisitos previos formales como es la formación académica reglada (Licenciatura o Grado en Psicología), estar colegiado, estar al corriente de la cuota colegial y no tener sanción deontológica, una formación y experiencia profesional específica y reconocida en Psicología del Deporte (acreditación de psicólogo del deporte del Consejo General de la Psicología de España) y experiencia aplicada en esports (al menos hasta que salga una formación de experto en esta área).

\section{Referencias}

Adamos, A. C., Beredo, J. D., Garcia, C. J. G., Mateo, W. B., Villalobos, M. J. M., Prado, S. V.,... Buenaventura, C. V. S. (2017). A comparison of brain activities stimulated by playing different video game genres using Electroencephalogram signal analysis. En 2017 IEEE 9th International Conference on Humanoid, Nanotechnology, Information Technology, Communication and Control, Environment and Management. Manila: Philippines.

Allen, M. S., Greenless, I. y Jones, M. (2013). Personality in sport: A comprehensive review. International Review of Sport and Exercise Psychology, 6(1), 184-208.

Allen, M. S., Magee, C. A., Vella, S. A. y Laborde, S. (2016). Bidirectional associations between personality and physical activity in adulthood. Health Psychology, 36, 332-336. https://doi. org/10.1037/hea0000371

Bányai, F., Griffiths, M., Király, O. y Demetrovics, Z. (2018). The psychology of esports: A systematic literature review. Journal of Gambling Studies. https://doi.org/10.1007/s10899-018-9763-1

Bean, A. M., Ferro, L. S., Vissoci, J. R. N., Rivero, T. y Groth-Marnat, G. (2016). The emerging adolescent World of Warcraft video gamer: A five factor exploratory profile model. Entertainment Computing, 17, 45-54. https://doi.org/10.1016/j.entcom.2016.08.006

Bediou, B., Adams, D. M., Mayer, R. E., Tipton, E., Green, C. S. y Bavelier, D. (2018). Meta-analysis of action video game impact on perceptual, attentional, and cognitive skills. Psychological Bulletin, 144(1), 77-110. https://doi.org/10.1037/bul0000130

Bertran, E. y Chamarro, A. (2016). Video gamers of League of Legends: the role of passion in abusive use and in performance. Adicciones, 28(1), 28. https://doi.org/10.20882/adicciones.787.

Billieux, J., Deleuze, J., Griffiths, M. D. y Kuss, D. J. (2015). Internet gaming addiction: The case of massively multiplayer online role-playing games. In N. el-Guebaly, G. Carra, y M. Galanter (Eds.), Textbook of addiction treatment: International perspectives (pp. 1515-1525). Nueva York, NY, Estados Unidos: Springer Milan.
Bonny, J. W. y Castaneda, L. M. (2017). Number processing ability is connected to longitudinal changes in multiplayer online battle arena skill. Computers in Human Behavior, 66, 377-387. https://doi.org/10.1016/j.chb.2016.10.005

Cantón, E. (2016). La especialidad profesional en Psicología del Deporte. Revista de Psicología Aplicada al Deporte y al Ejercicio Físico, 1, e12. https://doi.org/10.5093/rpadef2016a2

Carbonie, A., Guo, Z y Cahalane, M. (Junio de 2018). Positive personal development through esports. Twenty-Second Pacific Asia Conference on Information Systems, Japan. Recuperado de https://aisel.aisnet.org/pacis2018/125

Chang, Y.-H., Liu, D.-C., Chen, Y.-Q. y Hsieh, S. (2017). The Relationship between Online Game Experience and Multitasking Ability in a Virtual Environment. Applied Cognitive Psychology, 31, 653-661. https://doi.org/10.1002/acp.3368

Chiappe, D., Conger, M., Liao, J., Caldwell, J. L. y Vu, K.-P. L. (2013). Improving multi-tasking ability through action videogames. Applied Ergonomics, 44, 278-284. https://doi.org/10.1016/j. apergo.2012.08.002

Colzato, L. S., van den Wildenberg, W. P. M., Zmigrod, S. y Hommel, B. (2012). Action video gaming and cognitive control: playing first person shooter games is associated with improvement in working memory but not action inhibition. Psychological Research, 77, 234-239. https://doi.org/10.1007/s00426012-0415-2

Cox, R. H. (2009). Psicología del deporte: conceptos y sus aplicaciones (6 $6^{\mathrm{a}}$ ed.). Madrid, España: Editorial Médica Panamericana.

Deleuze, J., Christiaens, M., Nuyens, F. y Billieux, J. (2017). Shoot at first sight! First person shooter players display reduced reaction time and compromised inhibitory control in comparison to other video game players. Computers in Human Behavior, 72, 570-576. https://doi.org/10.1016/i.chb.2017.02.027

DEV (2016). Libro blanco del desarrollo español de videojuegos. Recuperado de http://www.dev.org.es/libroblancodev2016

Dindar, M., y Akbulut, Y. (2014). Motivational characteristics of Turkish MMORPG players. Computers in Human Behavior, 33, 119-125.

Funk, D., Pizzo, A. y Baker, B. (2018). Esport management: embracing esport education and research opportunities. Sport Management Review, 21(1), 7-13.

Fuster, H., Carbonell, X., Chamarro, A., y Oberst, U. (2013). Interaction with the game and motivation among players of massively multiplayer online role-playing games. The Spanish Journal of Psychology, 16, e43. https://doi.org/10.1017/sjp.2013.54

Gabbiadini, A., y Greitemeyer, T. (2017). Uncovering the association between strategy video games and self-regulation: A correlational study. Personality and Individual Differences, 104, 129-136. https://doi.org/10.1016/i.paid.2016.07.041

Gamerdic (2018). Términos que definen géneros y subgéneros de videojuegos. Recuperado de http://www. gamerdic.es/tema/generos

García-Naveira, A. (2018). Acreditaciones y coaching en los Colegios Oficiales de Psicología de España: estado de la cuestión. Informació Psicológica, 107, 47-65.

Gerber, A. (2017). esports and streaming: twitch literacies. Journal of Adolescent \& Adult Literacy, 67(3), 343-345. https://doi. org/10.1002/jaal.692

González-González, C., del Río, N. y Navarro-Adelantado, V. (2018). Exploring the Benefits of Using Gamification and Videogames 
for Physical Exercise: a Review of State of Art. International Journal of Interactive Multimedia \& Artificial Intelligence, 5(2), 46-52.

Grizzard, M., Tamborini, R., Lewis, R. J., Wang, L., y Prabhu, S. (2014). Being bad in a video game can make us more morally sensitive. $C y$ berpsychology, Behavior and Social Networking, 17, 499-504. https://doi.org/10.1089/cyber.2013.0658

Hallmann, K. y Giel, T. (2018). eSports - Competitive sports or recreational activity? Sport Management Review, 27(1), 14-20. https://doi.org/10.1016/j.smr.2017.07.011

Hamari, J. y Sjöblom, M. (2017). What is eSports and why do people watch it? Internet Research, 27(2), 211-232, https://doi. org/10.1108/IntR-04-2016-0085

Hilvoorde, I. V., y Pot, N. (2016). Embodiment and fundamental motor skills in esports. Sport, Ethics and Philosophy, 10(1), 14-27.

Hyun, G. J., Shin, Y. W., Kim, B.-N., Cheong, J. H., Jin, S. N., y Han, D. H. (2013). Increased cortical thickness in professional on-line gamers. Psychiatry Investigation, 10(4), 388-392. https://doi. org/10.4306/pi.2013.10.4.388

Kari, T., y Karhulahti, V.M. (2016). Do e-athletes move?: A study on training and physical exercise in elite e-sports. International Journal of Gaming and Computer Mediated Simulations, 8(4), 53-66.

Kokkinakis, A. V., Cowling, P. I., Drachen, A., y Wade, A. R. (2017). Exploring the relationship between video game expertise and fluid intelligence. PLOS ONE, 12(11), e0186621. https://doi. org/10.1371/journal.pone.0186621

Lee, B. W., y Leeson, P. R. C. (2015). Online gaming in the context of social anxiety. Psychology of Addictive Behaviors, 29(2), 473482. https://doi.org/10.1037/adb0000070

MAD Lions E.C. (2018). Club. Recuperado de https://madlions. $\mathrm{com} / \mathrm{club} /$

Meinz, E. J., Hambrick, D. Z., Hawkins, C. B., Gillings, A. K., Meyer, B. E., y Schneider, J. L. (2012). Roles of domain knowledge and working memory capacity in components of skill in Texas Hold'Em poker. Journal of Applied Research in Memory and Cognition, 1(1), 34-40. https://doi.org/10.1016/j.jarmac.2011.11.001

Morschheuser, B., Riar, M., Hamari, J., y Maedche, A. (2017). How games induce cooperation? A study on the relationship between game features and we-intentions in an augmented reality game. Computers in Human Behavior, 77, 169-183. https:// doi.org/10.1016/i.chb.2017.08.026

Newzoo (2017). Global games market report. Recuperado de https://newzoo.com/insights/articles/newzoos-key-developments-in-2017/

Newzoo. (2018). Global esports market report, Newzoo free. Recuperado de https://newzoo.com/insights/trend-reports/global-esports-market-report-2018-light/

Nuyens, F., Kuss, D.J., Lopez-Fernandez, O., y Griffiths, M.D. (2017). The experimental analysis of problematic video gaming and cognitive skills: a systematic review. Journal of Behavioral and Cognitive Therapy, 27, 110-117. https://dol.org/10.1016/j. itcc. 2017.05.001

ONTIER (2018). Guía legal sobre esports. Presente y futuro de la regulación de los esports en España. Recuperado de https://es.ontier.net/ia/guialegalesports-2018web.pdf

Pérez-Rubio, C., González, J., y Garcés de los Fayos, E.J. (2017). Personalidad y burnout en jugadores profesionales de e-sports. Cuadernos de Psicología del Deporte, 17(1), 41-50.
Peris-Delcampo, D. y Cantón, E. (2018). El perfil profesional del especialista en psicología del deporte en fútbol. Revista de Psicología Aplicada al Deporte y al Ejercicio Físico, 3, e9. https://doi. org/70.5093/rpadef2018a6

Powers, K. L., Brooks, P. J., Aldrich, N. J., Palladino, M., y Alfieri, L. (2013). Effects of video-game play on information processing: A meta-analytic investigation. Psychonomic Bulletin \& Review, 20, 1055-1079. https://doi.org/10.3758/s73423-013-0418-z

Puerta-Cortés, D. X., Panova, T., Carbonell, X., y Chamarro, A. (2017). How passion and impulsivity influence a player's choice of videogame, intensity of playing and time spent playing. Computers in Human Behavior, 66, 122-128. https://doi.org/10.1016/j. chb.2016.09.029

Railsback D. y Caporusso N. (2019). Investigating the Human Factors in eSports Performance. En Ahram T. (Ed), Advances in Human Factors in Wearable Technologies and Game Design. International Conference on Applied Human Factors and Ergonomics (AHFE 2018). Advances in Intelligent Systems and Computing, 795, 325-334. Springer, Cham. https://doi.org/10.1007/978-3379-94619-1_32

Richard, G., Mckinley, Z. y Ashley, R. (July 2018). Collegiate esports as learning ecologies: investigating collaborative learning and cognition during competitions. Conference in Proceedings of the 2018 Digital Games Research Association (DiGRA). Turín: Italy.

Ruiz-Barquín, R. y García-Naveira, A. (2013). Personalidad, edad y rendimiento deportivo en jugadores de fútbol desde el modelo de Costa y McCrae. Anales de Psicología, 29, 642-655.

Sala, G., Tatlidil, K. S., y Gobet, F. (2018). Video game training does not enhance cognitive ability: A comprehensive meta-analytic investigation. Psychological Bulletin, 144(2), 111-139. https:// doi.org/10.1037/bul0000139

Sánchez, A. y Remilllard, J. (2018). Esport: towards a hermeneutic of virtual sport. Cultura, Ciencia y Deporte, 38(13), 137-145.

Song, W. H., Han, D. H., y Shim, H. J. (2013). Comparison of brain activation in response to two dimensional and three dimensional on-line games. Psychiatry Investigation, 10(2), 115. https:// doi.org/10.4306/pi.2013.10.2.115

Stantion, R. (2015). A brief history of video games. Londres, Reino Unido: Robinson.

Taylor, T. (2012). Raising the stakes: e-sports and the professionalization of computer gaming. Cambridge, Reino Unido: The MIT Press.

Thompson, J. J., Blair, M. R., y Henrey, A. J. (2014). Over the hill at 24: persistent age-related cognitive-motor decline in reaction times in an ecologically valid video game task begins in early adulthood. PLOS ONE, 9(4), e94215. https://doi.org/10.1371/ journal.pone.0094215

Walsh, J., Barnes, J., Cameron, J., Goldfield, G., Chaput, J., Gunnell, K., Ledoux, A., Zemek, R. y Tremblay, M. (2018). Associations between 24 hour movement behaviours and global cognition in US children: a cross-sectional observational study. The Lancet Child \& Adolescent Health. Recuperado de https://www.sciencedirect.com/science/article/pii/S2352464218302785?via\%3Dihub. https://doi.org/10.1016/S2352-4642(18)30278-5

Wang, B., Taylor, L. y Sun, Q. (2018). Families that play together stay together: Investigating family bonding through video games. New Media \& Society, 20, 4074-4094. https://doi. org/10.1177/1461444818767667 
Weinberg, R.S. y Gould, D. (2010). Fundamentos de Psicología del Deporte y del Ejercicio Físico (4ª Edición). Madrid, España: Editorial Médica Panamericana.

Zhong, Z.-J. (2011). The effects of collective MMORPG (Massively Multiplayer Online Role-Playing Games) play on gamers' online and offline social capital. Computers in Human Behavior, 27(6), 2352-2363. https://doi.org/10.1016/j.chb.2011.07.014 